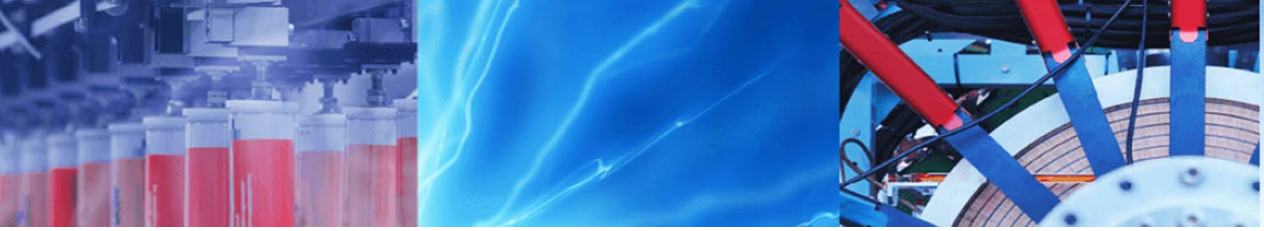

Research Article

\title{
Structural design of composite stiffened panel for a flat wing micro-aircraft
}

\author{
Xiuyang Qian ${ }^{1}$ (D) Yushan Zhou ${ }^{1} \cdot$ Menghui Wang $^{2} \cdot$ Liya Cai $^{1} \cdot$ Feng Pei $^{1}$
}

Received: 23 October 2019 / Accepted: 17 March 2020 / Published online: 20 March 2020

(c) Springer Nature Switzerland AG 2020

\begin{abstract}
In this study, the integral micro-sized composite wings are designed and manufactured by using carbon fiber reinforced plastics and low-density foam. The analytical expressions of bending stiffness of a multilayer sandwich structures for variable cross-section panel are determined. The obtained analytical bending stiffness is numerically verified using results of compressive buckling modes on wing panels from a numerical FE program and experimental tests by a series of stiffened structures which are empirically designed. Contrastive results demonstrate that the stiffener configuration tremendously affects the global buckling of the wing panels and the shapes, locations and intervals of the stiffeners which should be adjusted to construct a structure with maximum bending stiffness. Finally, with the use of the bi-directional evolutionary structural optimization method, the optimum design of the wing cross-section was determined by topology optimization method to pursue the best weight/stiffness. Compared with the initial structure, the optimum material layout of the topology structure performs better at reducing stress concentration and improving load carrying capacity of wing panels.
\end{abstract}

Keywords Composite material · Sandwich structure · Stiffener · Finite element model · Topology optimization

\section{Introduction}

Sandwich structures have got broad applications in many engineering sectors such as transportation vehicles and aircraft industry attribute to their high ratio of stiffness/ strength-to-weight [1]. They usually consist of two stiff exterior face-sheets and a low to moderate stiffness core as well as various supporting stiffeners. The composite structure has a considerably higher ratio of shear stiffness to weight than either of the equivalent beam made of only one material. However, the complex components bring more demands on manufacturing process. A lot of machined parts must be joined by adhesive bonding, co-curing or mechanical fastening [2]. This is particularly important in the aircraft industry. A large number of smaller parts such as skin panels, cores, ribs and stringers need to be joined together to fabricate as a single integral structure [3]. Fortunately, the increasing application of advanced composites gives the possibility and convenience to realize the weight saving potential and reduce the manufacturing costs and subsequent product maintenance costs in aircraft structures such as wing panels [4].

Structural design of an integral structure aircraft wing requires a layout of spars, ribs, and stringers based on the reference plane of the wing. Since the compressive bending performance of the wing panels is usually of great concern in this type of structure. Layout parameters such as shapes, intervals and location of stiffeners become very important on global buckling of the wing panel [5]. The spars, ribs and stringers could largely improve the bending stiffness of the panel. However, local buckling on the wing skin might occur before the wing panel reaches its own critical load if they are not appropriately arranged. Therefore, the stiffener shapes, shapes, intervals and location of

Xiuyang Qian, qianxiuyang@gacrnd.com | ${ }^{1} \mathrm{GAC}$ Automotive Research \& Development Center, Guangzhou 511434, China. ${ }^{2}$ SINOPEC LuoYang Engineering Co., LTD, Guangzhou 510620, China. 
stiffeners should be adjusted to construct a structure with optimized arrangement. So far, optimization design of the stiffened wing panels with different type of stiffeners has made significant progress. Composite wing panels with T-shaped [6] stiffeners had been optimized by using a twostep method to get an optimum super-stiffener design for the cross-sectional dimensions of the panel. HAT-stiffened [7] composite panels were also examined by a response surface methodology by Todoroki et al. They achieved a feasible optimal structure with low computational cost by optimizing the HAT-stiffener dimensions and the stacking sequences under buckling constraints. The other types of stiffeners such as $L, C, J$ and Z-stiffeners [8-11] has also been examined and optimized to obtain the minimal weight and maximum buckling loads for wing panels.

Additionally, the authors such as Guo et al. [12] investigated an aerobatic aircraft wing box and obtained a minimum weight optimal structure by optimizing the parameters of the wing skin and spar web. Monroy Aceves et al. [13] developed a material and design selection methodology using a small low air-speed wind turbine blade case which comprised uniform thickness shin and a unidirectional reinforcement. In terms of shin thickness and reinforcement area, a wide design space was made to select an optimum design. Kim et al. [14] applied a multilevel optimization approach to determine a structural optimization framework and the detailed cross-sectional parameters were presented. In the recent years, with the development of topology optimization technology and finite element method, the distributed structures have obtained more and more applications to the aeronautical industry [15-18]. Topology optimization technology has been proven to be of great useful for saving important weight amounts and improving buckling loads in recent aircraft designs. However, it is necessary to note that these optimal solutions were obtained in different cases with various constraints and engineering requirements and most investigations focused on the optimal design of wing panels with one type of stiffeners. When compared with the wing panels with different type of stiffeners, there is no properly standard to measure the comparison study by using different optimization methodologies and techniques.

This has motivated research into a micro-sized unmanned aircraft wing. The present investigations focus on the methodology development of the optimal design for stiffened composite wing panels based on the planning of experiments and emphasize on the close conformity of the developed finite element analysis for an unmanned flat wing micro-aircraft. In this study, integral composite wings are designed and manufactured by using ply continuity of composite laminates as the skin over the carbon fiber reinforced plastics (CFRP) stiffened construction and low density foam core. The low density foam is segmented and then coated with unidirectional carbon pre-preg in combination as various stiffeners such as $\mathrm{C}$-stiffener and I-stiffener. Thus, the supporting stiffeners are assembled and inserted inside the skin framework. Based on the empirical design, the material distribution of the wing cross-section is determined by topology optimization method aiming at obtaining the optimal distributed structure of the stiffeners. The analytical expressions of bending stiffness of a variable cross-section sandwich structure give the properly standard to measure the optimal solutions obtained for the integral structure wings with different type of stiffeners.

\section{Theoretical and numerical methods}

It is well known that accurately predicting the bending behavior of a sandwich structure with embedded stiffeners [19] is a challenging problem. As shown in Fig. 1, a coupling action of vertical bending and shear would be induced for sandwich structures under three-point bending condition. Allen [20] gave the total deflection at the mid-point of a sandwich beam under a transverse load $P$ as following:

$\delta=\frac{P L^{3}}{48(E I)_{e q}}+\frac{P L}{4(G A)_{e q}}$

where $(E I)_{e q}$ is the equivalent flexural rigidity:
Fig. 1 Sketch of sandwich beam under three-point bending condition

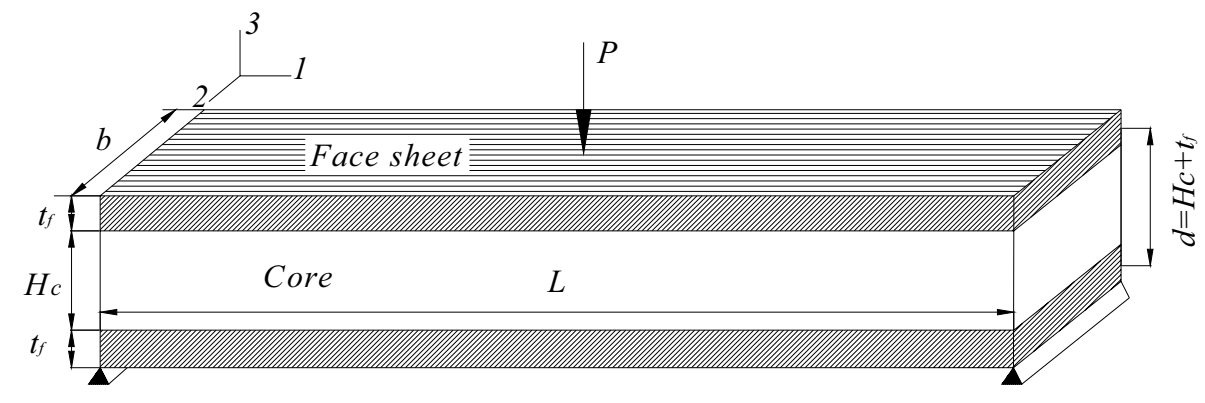




$$
\begin{aligned}
(E I)_{e q} & =\left(E_{f} I_{f}\right)_{e q}+\left(E_{c} I_{c}\right)_{e q}+\left(E_{f} l\right)_{e q} \\
& =\frac{E_{1}^{f} b t_{f}^{3}}{6}+\frac{E_{c} b H_{c}^{3}}{12}+\frac{E_{1}^{f} b t_{f} d^{2}}{2}
\end{aligned}
$$

$(G A)_{e q}$ is the equivalent shear rigidity dictated by the shear stiffness of the core:

$(G A)_{e q}=\frac{G_{c} b d^{2}}{H_{c}}$

where $b$ and $L$ are the width and length of the sandwich beam; $t_{f}$ and $H_{c}$ are thickness of face-sheets and supporting core; $E_{f} G_{f}$ and $E_{c^{\prime}} G_{c}$ are the elastic modulus and shear modulus of the face-sheets and the core respectively; $\left(E_{f} f_{f}\right)_{e q}$ and $\left(E_{c} l_{c}\right)_{e q}$ are the equivalent flexural rigidity of the face-sheets and core on themselves' centroids; $\left(E_{f}\right)_{e q}$ is the flexural rigidity of the face-sheets on the whole sandwich beam's centroid. In terms of elastic modulus $E_{1}^{f}$ of the facesheets in the 1-direction is defined as in Fig. 1.

In this work, we discuss the multilayer sandwich structures in variable cross-section wing panel applied to an integral structure flat wing micro-aircraft as shown in Fig. 2.

As the profile area of the sandwich beam changes in linear fashion, the width $b$ of the sandwich beam and thickness $H_{c}$ of the core may be equivalent to $b^{*}$ and $H_{c}^{*} . b^{*}$ and $H_{c}{ }^{*}$ are width and thickness on centroid of the sandwich beam. Perfect bonding is also assumed to address this issue [21].

The equivalent flexural rigidity $(E I)_{e q}$ and shear rigidity $(G A)_{e q}$ of the variable cross-section sandwich beam may be defined as:

$$
\begin{aligned}
& (E I)_{e q}=\left[\left(E_{f} I_{f}\right)_{e q}+\left(E_{c} I_{c}\right)_{e q}+\left(E_{f}\right)_{e q}+\left(E_{f} I_{s}\right)_{e q}\right] \cos \alpha \\
& =\frac{E_{1}^{f} b^{*} t_{f}^{3}}{6}+\frac{E_{c} b^{*} H_{c}^{* 3}}{12}+\frac{E_{1}^{f} b^{*} t_{f}\left(H_{c}^{*}+2 t_{f}\right)^{2}}{2}+E_{1}^{f} l_{s}^{*} \\
& (G A)_{e q}=\left[\left(G_{c} A_{c}\right)_{e q}+\left(G_{s} A_{s}\right)_{e q}\right] \cos \alpha=\frac{G_{c} b^{*}\left(H_{c}^{*}+2 t_{f}\right)^{2}}{H_{c}^{*}}+G_{13}^{f} A_{s}^{*}
\end{aligned}
$$

where $I_{s}^{*}$ is the moment of inertia of stiffener on the centroids; $A_{s}{ }^{*}$ is the profile area of stiffener on their centroids;
$G_{13}{ }^{f}$ is the of the shear modulus of the stiffener in the 1,3-direction; $a$ is the variable angle as illustrated in Fig. 2 . The flexibility $\lambda$ of variable cross-section sandwich beam reinforced by CFRP stiffeners can be constructed from Eqs. (1), (4) and (5).

$$
\begin{aligned}
\lambda= & \frac{\delta}{P}=\frac{L^{3}}{48(E I)_{e q}}+\frac{L}{4(G A)_{e q}} \\
= & \frac{L^{3}}{48\left[\frac{E_{f}^{f} b^{*} t_{f}^{3}}{6}+\frac{E_{c} b^{*} H_{c}^{* 3}}{12}+\frac{E_{1}^{f} b^{*} t_{f}\left(H_{c}^{*}+2 t_{f}\right)^{2}}{2}+E_{1}^{f} l_{s}^{*}\right]} \\
& +\frac{L}{4\left[\frac{G_{c} b^{*}\left(H_{c}^{*}+2 t_{f}\right)^{2}}{H_{c}^{*}}+G_{13}^{f} A_{s}^{*}\right]}
\end{aligned}
$$

The elastic bending stiffness $K$ of the variable crosssection sandwich beam is governed by:

$\kappa=\frac{P}{\delta}=1 / \lambda$

Here, the influence of stiffener's structure and dimension in the stiffness of the sandwich beam is integrated in terms of the moment of inertia $l_{s}{ }^{*}$ and profile area $A_{s}{ }^{*}$. This gives the possibility to compare the compressive buckling modes of the composite wing panels with different type of stiffeners.

For numerical simulation, ABAQUS is a well developed commercial Finite Element Modeling (FEM) tool that has been employed in such an investigation for its applicability to this problem. A virtual test was modeled by ABAQUS as shown in Fig. 3. The laminates were modeled using the Composite Layup Function available within ABAQUS and 8-node doubly curved thick shell elements SC8R were used. Low density foam was defined as isotropic elastic solid and 20-node quadratic brick solid elements C3D20R were used. The composite wing was subjected to the compressive loading by a rigid indenter with displacement-controlled [22]. The compressive loading was applied to a reference point appointed on the indenter and interaction between the lower surface of the indenter and the upper center of the wing surface are correspondingly constrained. Corresponding displacement and reaction force on the rigid indenter could be conveniently output to this reference point
Fig. 2 Sketch of a variable cross-section sandwich beam under three-point bending condition

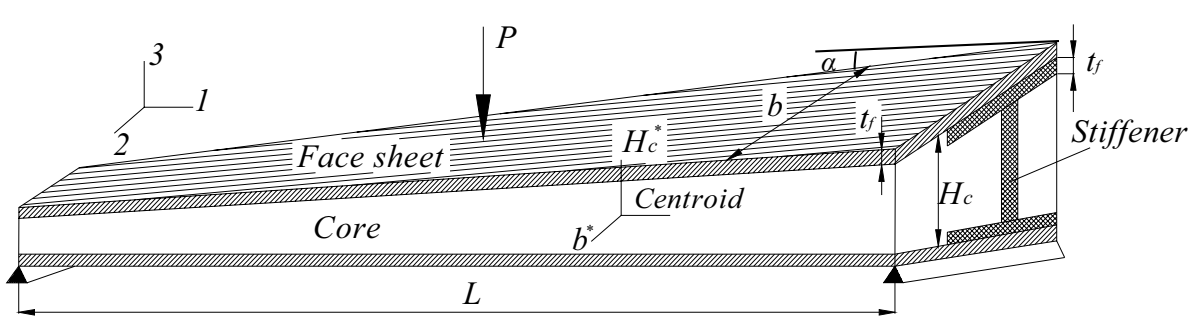

SN Applied Sciences A SPRINGER NATURE journa 
Fig. 3 Schematic diagram of the FE modeling

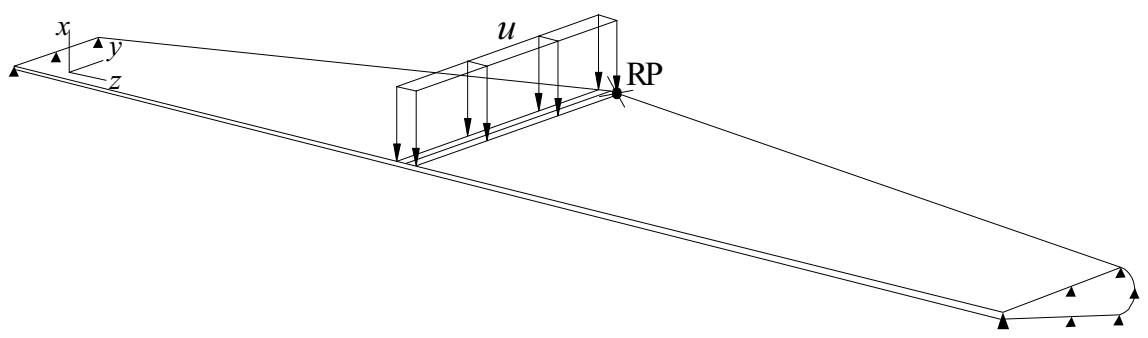

Boundary conditions

Fig. 4 Configuration and dimension of wing model (length units: $\mathrm{mm}$ )

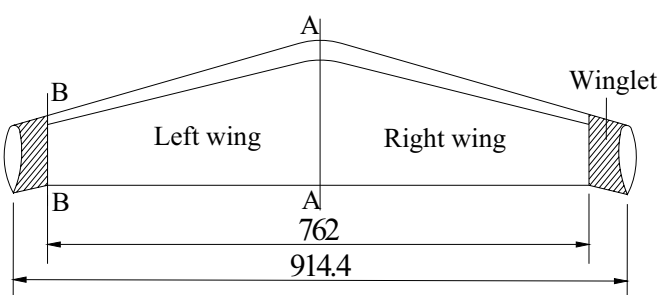

(a) The whole dimension

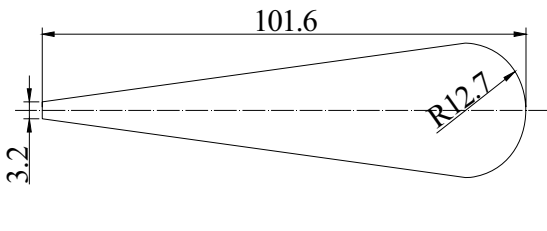

(b) A-A section

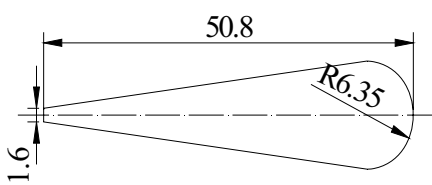

(c) B-B section

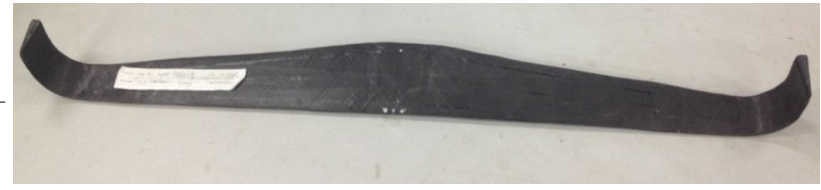

(d) Physical model
Table 1 Stacking sequence of the wing

\begin{tabular}{ll}
\hline Location of the wing & Stacking sequences \\
\hline Skin & $(0 / 45 /-45 / 45 /-45 / 90)$ \\
Stiffener & $(45 /-45 / 45 /-45 / 45 /-45)$ \\
Winglet & $(0 / 45 /-45 / 0)$ \\
\hline
\end{tabular}

when the compressive loading applied. The boundary conditions were also shown in Fig. 3.

\section{Experimental design and wing panel examples}

\subsection{Wing description}

The outer geometry of the flat wing is illustrated in Fig. 4. Figure 4a shows the dimension of the whole wing and Fig. $4 b, c$ show the dimensions of $A-A$ section and $B-B$ section. The physical model of the wing sample is given in Fig. $4 \mathrm{~d}$. The stacking sequences of the unidirectional laminates are quasi-isotropic $(0 / \pm 45 / 90)$ as shown in Table 1. And the properties of the unidirectional carbon pre-preg and foam used in our experiments are provided in Table 2. Figure 5 gives the uniaxial tension and compression stress-strain curves for the foam and it will be utilized as an input for the foam in finite element analysis software ABAQUS.

There are four different cross sections of composite wing in the experiment, including skin-foam, skin-stringer, $\mathrm{C}$-beam and I-beam structure. The schematics of the wing and stiffener profiles are shown in (a), (b), (c) and (d) of Fig. 6 . The three point bending test was performed to verify the load carrying capacity of the wings as shown in Fig. 7.

\subsection{Results of the structural analysis}

Load carrying capacity is closely related to the elastic bending stiffness of the sandwich beams, and shows a significantly positive correlation. Here, $K_{\text {the }}$ is the theoretical elastic bending stiffness of the sandwich beams as formulated in Eqs. (6)-(7). Generally, the elastic modulus, shear modulus and Poisson ratio are known just for the unidirectional lamina. However, multilayer composites are mainly used in the engineering. So the elasticity constants of the multilayer laminate could be obtained on the basis of classical lamination theory [23] as following. Quasi isotropic laminated structure is used in this study.

\section{SN Applied Sciences}


Table 2 Properties of unidirectional prepreg and foam

\begin{tabular}{|c|c|c|c|}
\hline \multicolumn{2}{|l|}{ Unidirectional prepreg } & \multicolumn{2}{|l|}{ Foam } \\
\hline Properties & Value & Properties & Value \\
\hline $0^{\circ}$ Tensile modulus (GPa) & 140 & Elastic modulus (MPa) & 70 \\
\hline $90^{\circ}$ Tensile modulus (GPa) & 7.1 & Shear modulus (MPa) & 19 \\
\hline Poisson's ratio & 0.28 & Tensile strength (MPa) & 1.9 \\
\hline In-plane shear modulus (GPa) & 4.5 & Compression strength (MPa) & 0.9 \\
\hline Interlayer shear modulus (GPa) & 3.6 & Poisson's ratio & 0.37 \\
\hline $0^{\circ}$ Tensile strength $(\mathrm{MPa})$ & 1385 & Volume density $\left(\mathrm{kg} / \mathrm{m}^{3}\right)$ & 52 \\
\hline $0^{\circ} \mathrm{Compression}$ strength (MPa) & 950 & & \\
\hline Interlayer shear strength (MPa) & 62.5 & & \\
\hline Ply thickness (mm) & 0.122 & & \\
\hline Resin volume content (\%) & 52 & & \\
\hline Area density $\left(\mathrm{g} / \mathrm{m}^{2}\right)$ & 187 & & \\
\hline
\end{tabular}

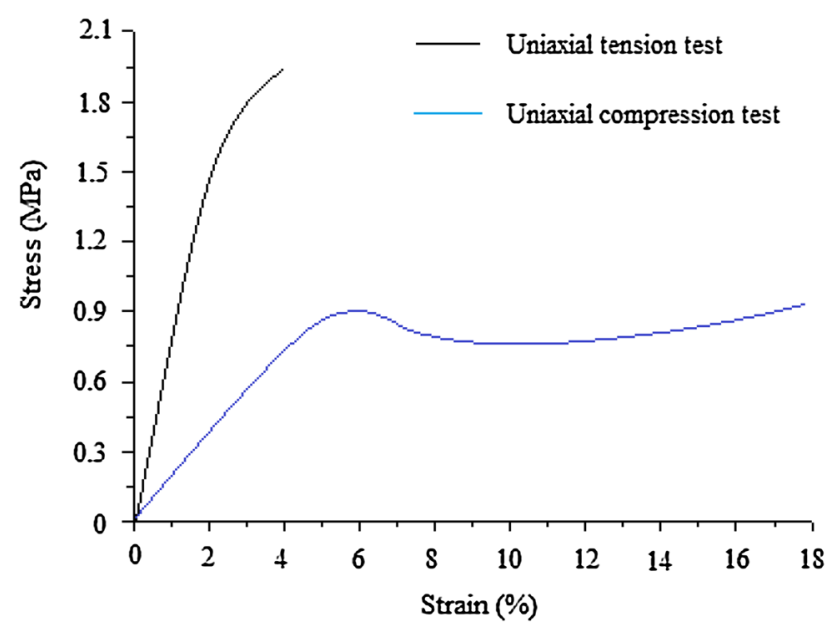

Fig. 5 Uniaxial tension and compression stress-strain curves for the foam
For symmetrical multidirectional laminate, the force is only applied in the plane. So the stress-strain relationship of the $K$-layer unidirectional lamina in the $x, y$ coordinate systems is written as:

$\left[\sigma_{x}\right]^{K}=[\bar{Q}]^{K}\left[\varepsilon_{x}\right]$

where $[\bar{Q}]^{K}$ and $\left[\sigma_{x}\right]^{K}$ are the off-axis stiffness matrix and stress of the $K$-layer unidirectional lamina in $x, y$ coordinate systems.

While a parallelepiped with an in-plane size of unit width $1 \times 1$ and a height of laminate thickness $t$ is taken out from the $\mathrm{N}$-layer laminate. The stress-strain relationship can be obtained by using the following equation:

$[N]=\sum_{K=1}^{N} \int_{z_{K-1}}^{z_{K}}[\bar{Q}]^{k}[\varepsilon] d z=\sum_{K=1}^{N}[\bar{Q}]^{k}\left(z_{K}-z_{K-1}\right)[\varepsilon] d z=[A][\varepsilon]$
Fig. 6 Cross section schematic of four stiffened structures (length units: $\mathrm{mm}$ )

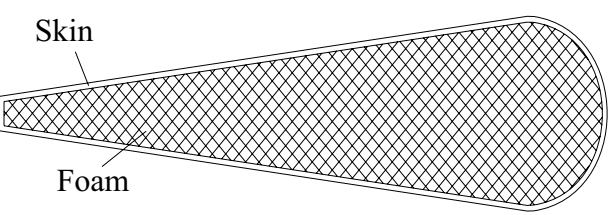

(a) Skin-foam

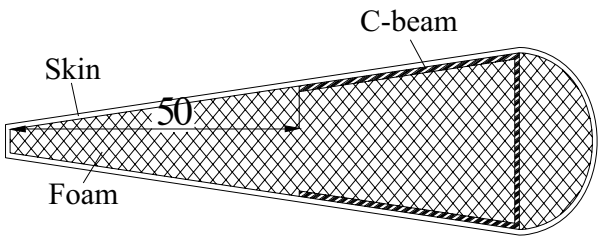

(c) C-beam

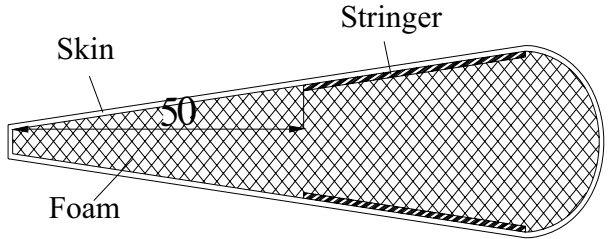

(b) Skin-stringer

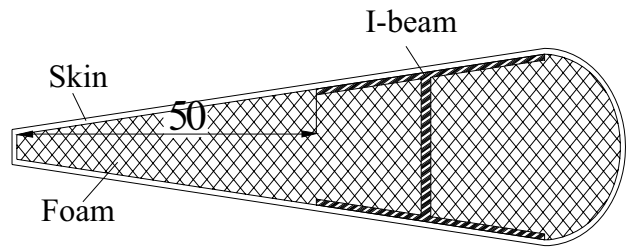

(d) I-beam 


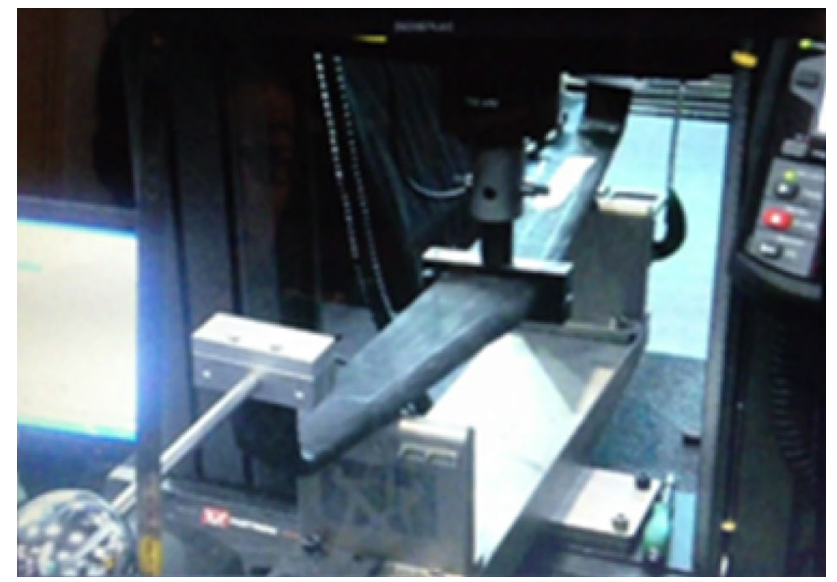

Fig. 7 The three-point bending test setup

where $z_{K}-z_{K-1}$ is the thickness of the $K$-layer unidirectional lamina; $[A]$ is the matrix of elastic stiffness coefficient. And

$A_{i j}=\sum_{K=1}^{N}[\bar{Q}]_{i j}^{K}\left(z_{K}-z_{K-1}\right)$

When taking the inverse of the matrix $[A]$, the third order compliance matrix $[a]=[A]^{-1}$ can be obtained. Where $a_{\mathrm{ij}}(\mathrm{i}, \mathrm{j}=1,2,3)$ is the compliance constant. So the strain is obtained as:

$[\varepsilon]=[a][N]$

The section area of the laminate is $1 \times t=t$, thus, the elasticity constants of the laminate could be obtained as:

$\left\{\begin{array}{l}E_{1}=\frac{1}{a_{11} t} \\ E_{2}=\frac{1}{a_{22} t} \\ G_{12}=G_{13}=\frac{1}{a_{33} t} \\ v_{12}=-\frac{a_{12}}{a_{11}}\end{array}\right.$

where $E_{1}$ and $E_{2}$ are the elastic modulus in $x$ and $y$ directions; $G_{12}$ and $G_{13}$ are the shear modulus in $x y$ and $x z$ directions; $v_{12}$ is the Poisson ratio of unidirectional lamina in $x y$ directions.

Then combined with the geometry and dimension of the flat wing in Fig. 4 and properties of unidirectional prepreg and foam in Table 2, the theoretical elastic bending stiffness can be written as:

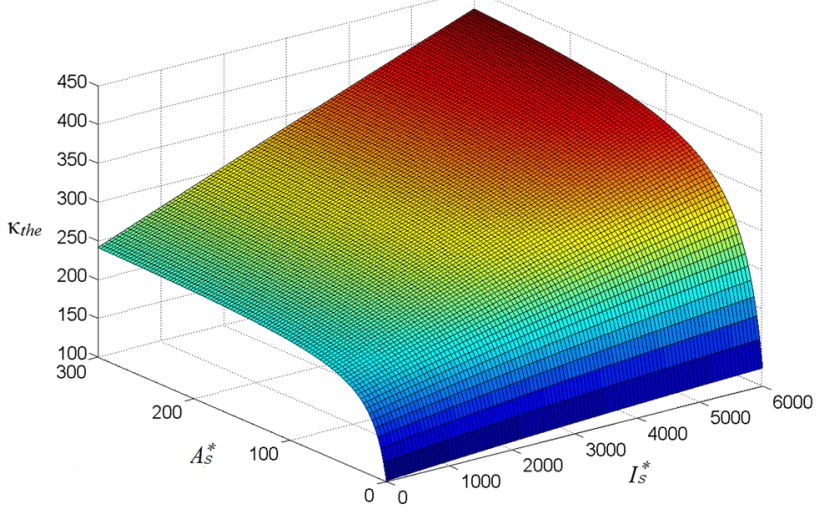

Fig. 8 The theoretical prediction model of the bending stiffness

$\kappa_{\text {the }}=1 /\left(\frac{29.76}{l_{s}^{*}+7415.6}+\frac{0.04}{A_{s}^{*}+6.8}\right)$

It was depicted in Fig. 8, the plot showed some extent of nonlinearity. It followed from Fig. 8 that $K_{\text {the }}$ increased sharply as $A_{s}{ }^{*}$ increased in a certain range and then had a slowly increase. However, $I_{s}^{*}$ had a linear influence on $K_{\text {the }}$ all the way. Based on Eq. (13), the bending stiffness of sandwich beams can be directionally predicted with different stiffeners.

From the experiments, the elastic bending stiffness $K_{\text {exp }}$ can be estimated by:

$\kappa_{\exp }=\frac{\Delta P}{\Delta \delta}$

where $\Delta P$ and $\Delta \delta$ are the increments of the load and the displacement of the indenter, respectively. It was illustrated as shown in Fig. 9.

The finite element modeling (FEM) of the wing structures with four kinds of cross sections in Fig. 6 were implemented, respectively. The comparisons of load versus deflection curves were obtained from simulations in the FEM and theoretical prediction from Eq. (9) as shown in Fig. 9. When compared with the experimental results, the results predicted by the FEM prove a good accordance to the experimental data. However, the theoretical predictions of the elastic bending stiffness were generally a little lower than the corresponding experimental results except skin-foam structure. One possible reason is that the theoretical expressions did not take into account the interaction between the stiffeners and the foam core. Moreover, the adhesive which partially filled the foam core could also lead to a stiffening effect [21].

\section{SN Applied Sciences}



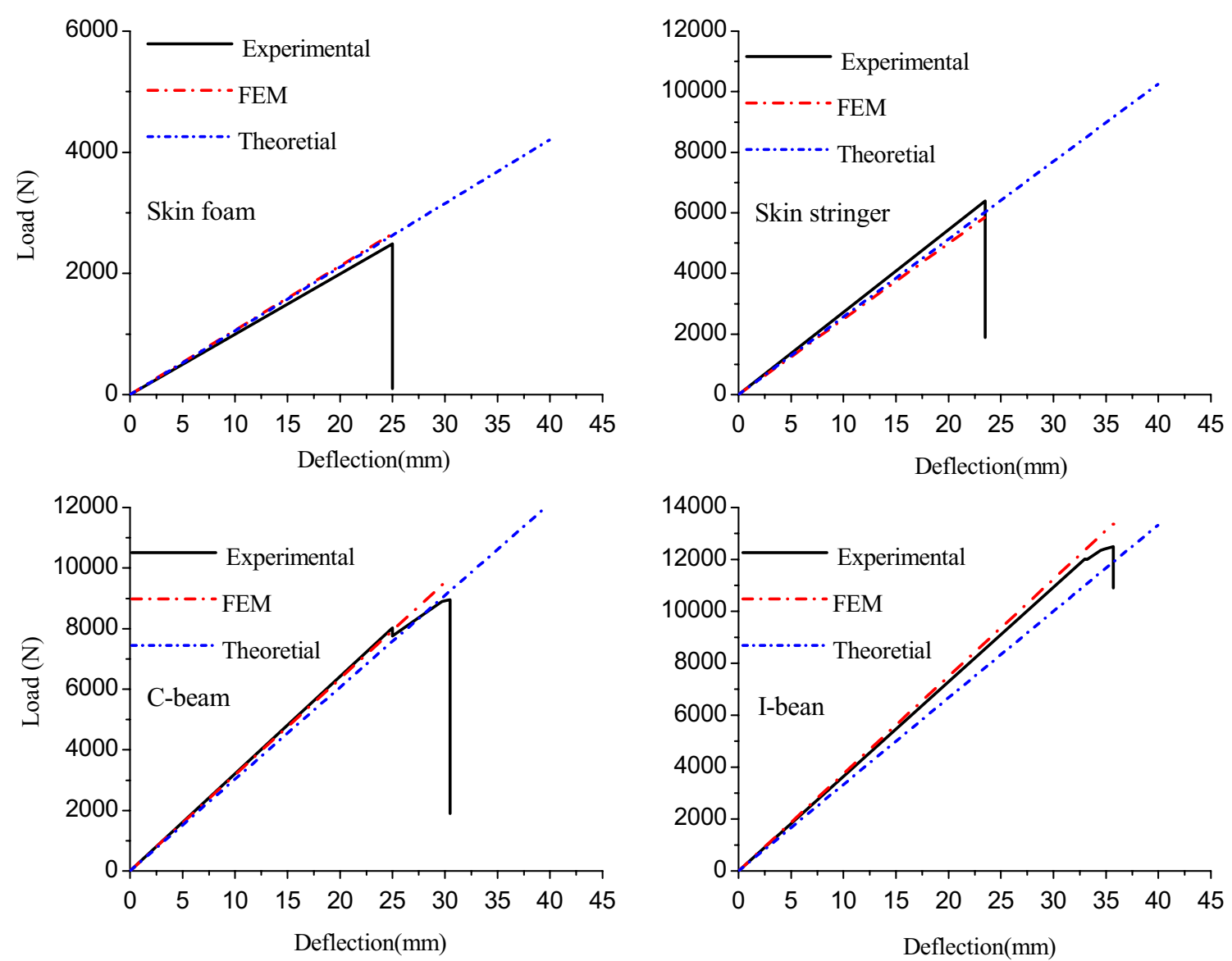

Fig. 9 Comparison of the force versus deflection curves of different stiffened wings

\section{Analysis of wing panel with a combinatorial stiffener}

In this section, a combinatorial stiffener for the wing panel is proposed in order to enhance the load carrying capacity of the composite wing. First, the properties of the stiffener as well as its design and manufacturing were introduced. Then, the numerical and theoretical methods were used to verify the improvements of the combinatorial structure in contrast to the conventional structures in Sect. 3.

\subsection{Structure description}

Figure 10a shows the structure schematic of the composite wing. The low density foam is segmented as shown in Fig. 11 and then coated with carbon pre-preg. The outstanding property of the proposed structure is originated from the stiffener inserted inside the composite wing
Fig. 10 Cross section schematic of the wing panel and its stiffener (length units: $\mathrm{mm}$ )

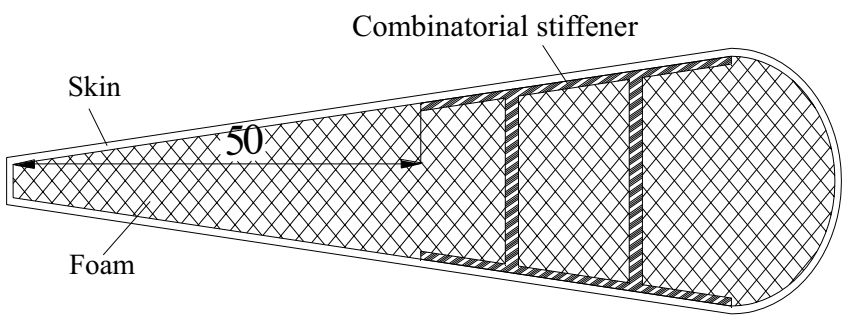

(a) Cross section

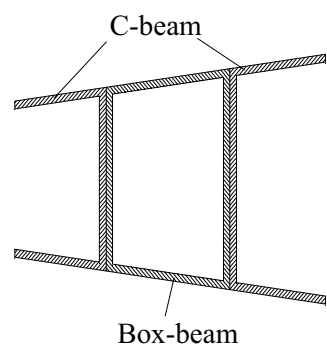

(b) Stiffener 
Fig. 11 Segmentation image of the low density form core

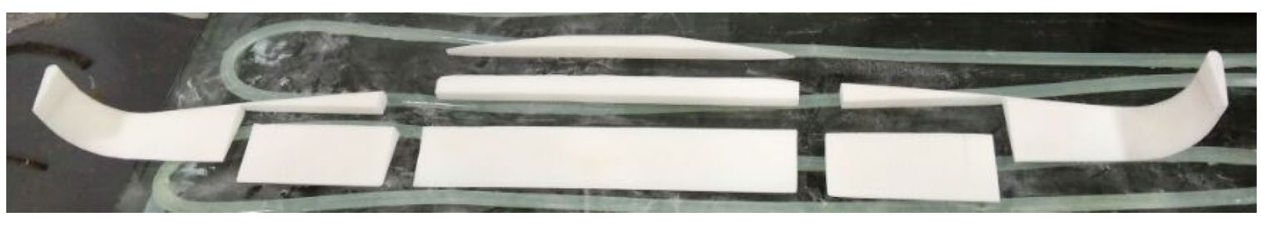

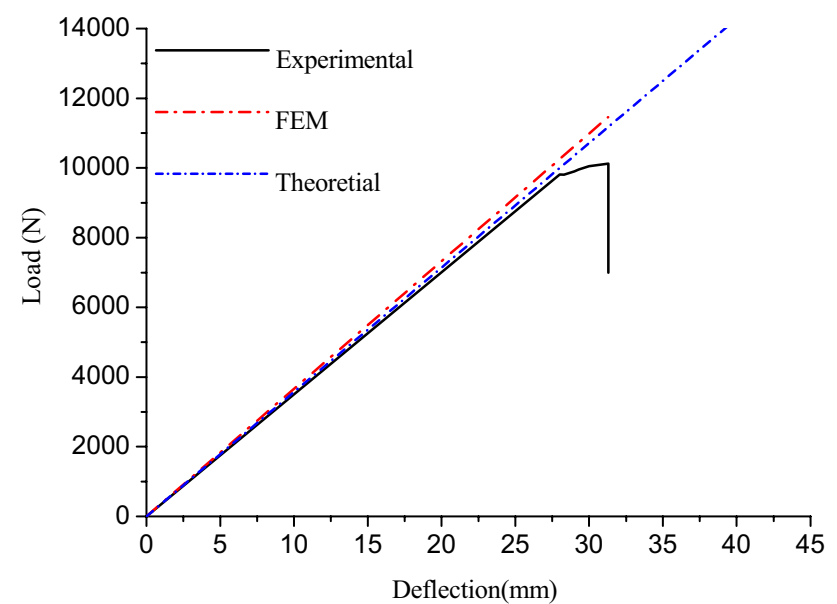

Fig. 12 Comparison of load versus deflection cures of the combinatorial stiffener

skin, including two C-beams back-to-back, and a box beam inserted between the two C-beams. This combinatorial stiffener is made from carbon pre-preg as shown in Fig. 10b. Then, the stiffener and low density foam are assembled into an entire composite wing core with epoxy adhesive. The stacking sequence is the same as the conventional structure wings as shown in Table 1.

\subsection{Analysis results of the combinatorial stiffener}

The load versus deflection curves predicted by FEM and theoretical model of the combinatorial stiffened structure wing are compared with the experimental test as shown in Fig. 12. A good agreement was obtained the numerical solution and the experimental data. However, the experimental result was lower than numerical analysis. One possible reason is that the structure is broken into a finite number of elements in the finite element modeling, which reduces the number of degrees of freedom and stiffens. Therefore, it is expected to see a larger stiffness in FEM.

The elastic bending stiffness of the different structure wings are summarized in Table 3 , in which $K_{\text {exp }}, K_{F E M}$ and $K_{\text {the }}$ were the experimental, FEM and theoretical elastic bending stiffness of the composite wings, respectively. It can be seen from Table 3 that the modular structure showed a reasonable improvement on load carrying capacity of the wing in contrast to the conventional structures.
Table 3 Summary of the results for different structural stiffeners

\begin{tabular}{lcll}
\hline Stiffener structures & $\kappa_{\text {exp }}(\mathrm{N} / \mathrm{mm})$ & $\kappa_{\text {the }}(\mathrm{N} / \mathrm{mm})$ & $\kappa_{\text {FEM }}(\mathrm{N} / \mathrm{mm})$ \\
\hline Skin-foam & 99.5 & 102.2 & 106.1 \\
Skin-stringer & 283.9 & 277.8 & 249.4 \\
C-beam & 310.3 & 303 & 317.5 \\
I-beam & 347.1 & 331.1 & 354.5 \\
Combinatorial stiffener & 350.4 & 357.1 & 366.4 \\
\hline
\end{tabular}

In addition to the results mentioned above, the damage evolution characterized by the distribution of shear stress on the composite skin was displayed by the graphical illustration (see, Fig. 13). As can be observed from Fig. 13, the failure (over the ultimate shear strength according to the Maximum Stress Criterion) had initiated at the upper center span where shear stress concentration was the highest and propagated at the angle of $45^{\circ}$. The damaged areas were shown in the lighter colors by the finite elements analysis. At the exactly zone, damage was appeared in the experimental test as shown in Fig. 13. In particular, it was clear in Fig. 13 that the upper skin (loading area) of the wing had damaged by shear delamination and the crack was also propagated at the angle of $45^{\circ}$. The finite element analysis performed in this study matched well with the results obtained from the experiment.

\section{Topology optimization of the wing}

It should be indicated that the structures discussed above are samples through our empirical design. In order to further improve the load carrying capacity of the integral composite wing with supporting stiffeners, the technology of topology optimization should be introduced to design the supporting structures of the wing cross-section.

Among current topology, the concept of gradually removing inefficient material from a structure plays a significant role. Xie et al. [24] presented an evolutionary structural optimization (ESO) method based on the simple concept as early as 1993. Later, they proposed bi-directional evolutionary structural optimization (BESO) method as an improved algorithm. Based on the BESO method, materials could be removed and added simultaneously in accordance with the sensitivity numbers $[25,26]$. However, the void elements, as the sensitivity numbers, are difficult to be estimated and 


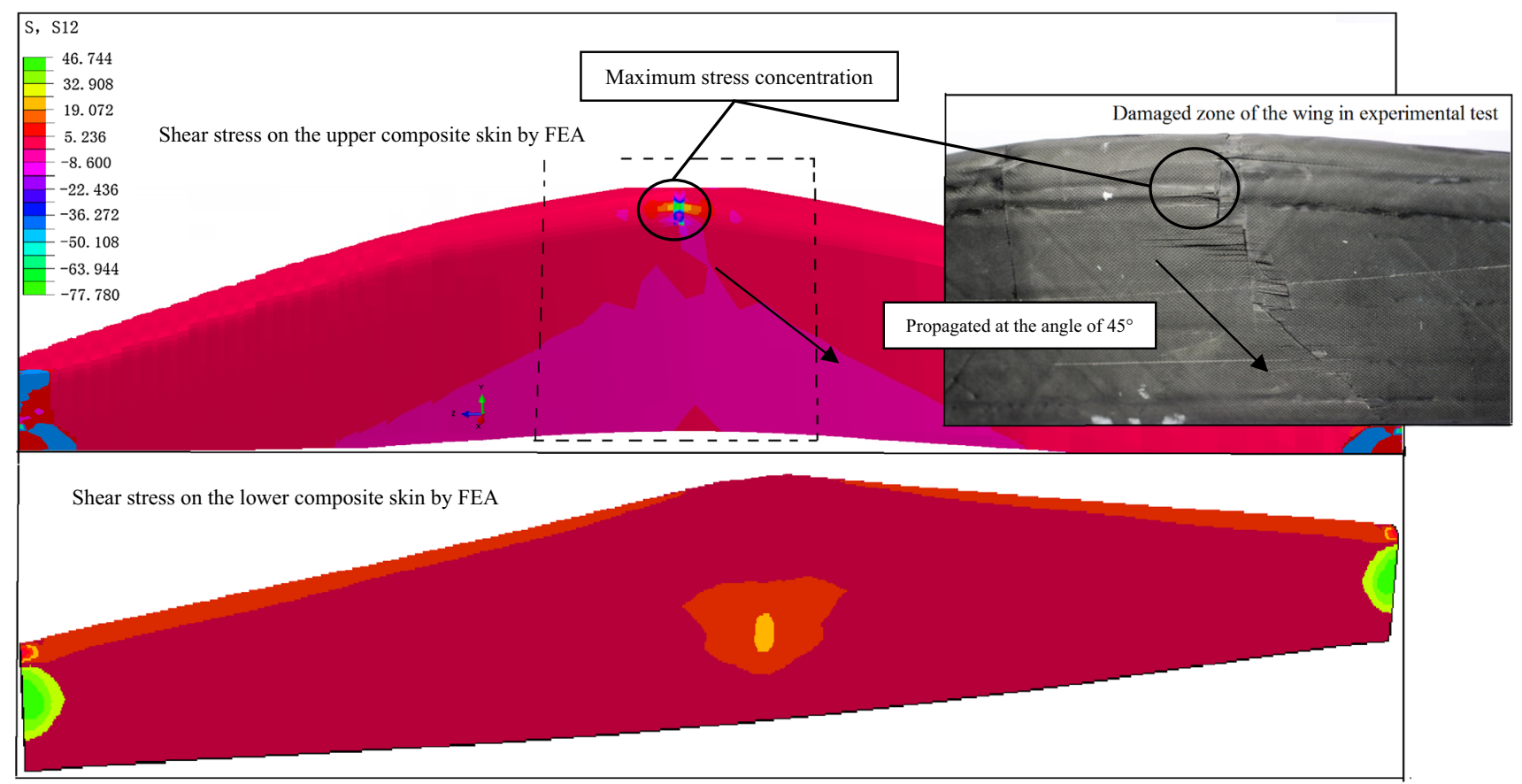

Fig. 13 Comparison of the results by FEA and experimental test

they are not included in the finite element analysis due to the absence of information available for void elements. Fortunately, Huang and Xie [27] developed a soft-kill BESO method by using the material interpolation scheme with penalization to solve this problem. In this work, the soft-kill BESO method was used to determine the optimum structure of the wing cross-section.

\subsection{Topology optimization method}

The objective of topology optimization for a continuum structure is usually devoted to finding the stiffest structure and minimizing the weight compliance with a given volume of material. So the formulation of topology optimization [28] can be generally written as:

Find $b_{j} ; j=1, \ldots, N$

Minimize $\quad C=\frac{1}{2} f^{T} u$

Subject to $K(b) u=f$

$$
\begin{aligned}
& \sum_{j=1}^{N} V_{j} b_{j} \leq V^{*} \\
& b_{j}=b_{\min }>0 \text { or } 1
\end{aligned}
$$

where $b, f, u$ and $C$ are the design variable vector, the applied load vectors, the applied displacement vectors and the mean compliance, respectively. $N$ is the total number of elements in this system. $V_{j}$ denotes the volume of the jth element and $V^{*}$ is the prescribed total structural volume. The design variables $b_{j}$ is used to declare the absence ( 0 ) and presence ( 1 ) of an element. $b_{\min }$ namely the lower bound of the design variables, is endowed with the teeny value to avoid the singular matrix.

In the soft-kill BESO method, the discrete design variables with two bound materials are generally used. Therefore, the sensitivity number can be calculated by relative ranking of the sensitivity of an individual element as:

$\alpha_{j}^{e}=-\frac{1}{p} \frac{\partial C}{\partial b_{j}}=\left\{\begin{array}{lll}\frac{1}{2} u_{j}^{T} k_{j}^{0} u_{j} & \text { when } & b_{j}=1 \\ \frac{b_{\min }^{p-1}}{2} u_{j}^{T} k_{j}^{0} u_{j} & \text { when } & b_{j}=b_{\min }\end{array}\right.$

where $k_{j}^{0}$ is the stiffness matrix of the solid element. $u_{j}$ is the corresponding displacement vector and $p$ is the penalty exponent.

\subsection{Optimization results of the wing cross-section}

In this case, we adopt the soft-kill BESO method to optimize the wing cross-section. In Fig. 14, the design domain is discretized with 22,525 four node plane stress elements. Also, the boundary conditions of the wing cross section are illustrated in the figure, wherein, the leading 
and trailing edges are clamped. First, we suppose that the whole wing is made from carbon pre-preg and then the soft-kill BESO method is implemented to remove the inefficient materials from the structure. In terms of the material properties, Shear modulus of $4.5 \mathrm{GPa}$ and Poisson's ratio of 0.28 are assumed (as shown in Table 2). Finally, only $40 \%$ of the material could be available in the final result, meaning that the weight of the optimized structure will be reduced by $60 \%$.

Figure 15 gives the optimum results obtained from the soft-kill BESO method. From Fig. 15, the optimum stiffener presents a little tunnel distribution consisting of multiple rib positions and flexible spars. In Fig. 16, the stress distribution of the initial wing cross-section under imposing force of $200 \mathrm{~N}$ is illustrated. From Fig. 16, it is apparent that the stress concentration is occurred around the leading and trailing edges due to the inhomogeneous stress distribution, and the maximum von Mises stress is 635.54 MPa. Then, for the optimization result whose stress distribution is shown in Fig. 17, the maximum von Mises stress is reduced to $167.45 \mathrm{MPa}$ and there is no apparent stress concentration occurred in the optimized structure. On the basis of the comparison mentioned above, it can be revealed the technology of topology optimization effectively improves the buckling stiffness of wing panels and reduce the stress concentration for the wing cross section.

$I_{s}^{*}$ and $A_{s}^{*}$ of the optimal stiffened structure in Fig. 16 were calculated in the CAD software, then were substituted into Eq. (13). By the theoretical equivalent model presented in Sect. 2, the theoretical buckling stiffness of the optimal stiffened structure can be obtained. Results show that the theoretical buckling stiffness of the wing would be up to $502.9 \mathrm{~N} / \mathrm{mm}$. Compared with the data in Table. 3, the load capacity of the wing panels is largely increased than those empirical designs. Finally, for the issues of fabricating process, the rapid development of the additive manufacturing (3D printing) makes it possible to fabricate the complicate structures yielded from
Fig. 14 Boundary conditions of the wing cross-section edge

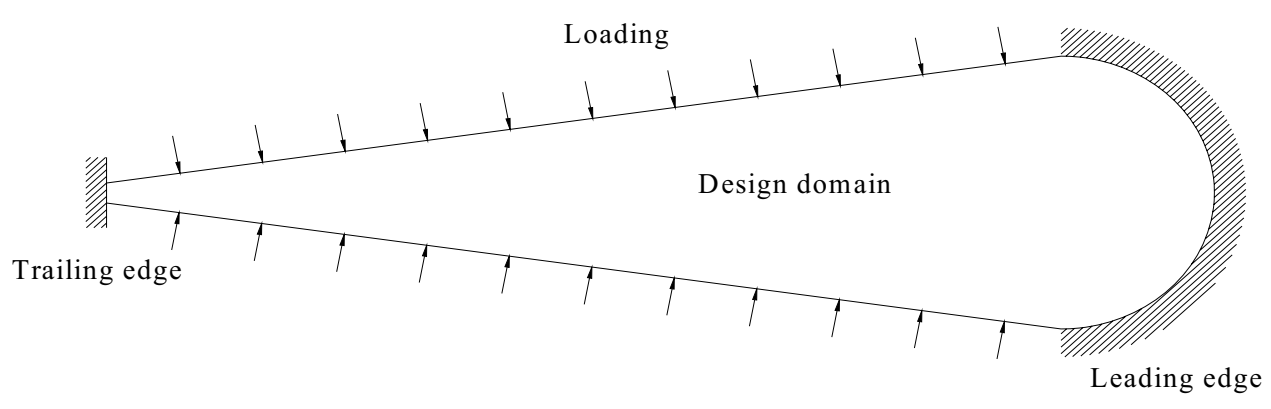

Fig. 15 The optimum layout of the stiffened structure

Fig. 16 Stress distribution of initial wing cross-section
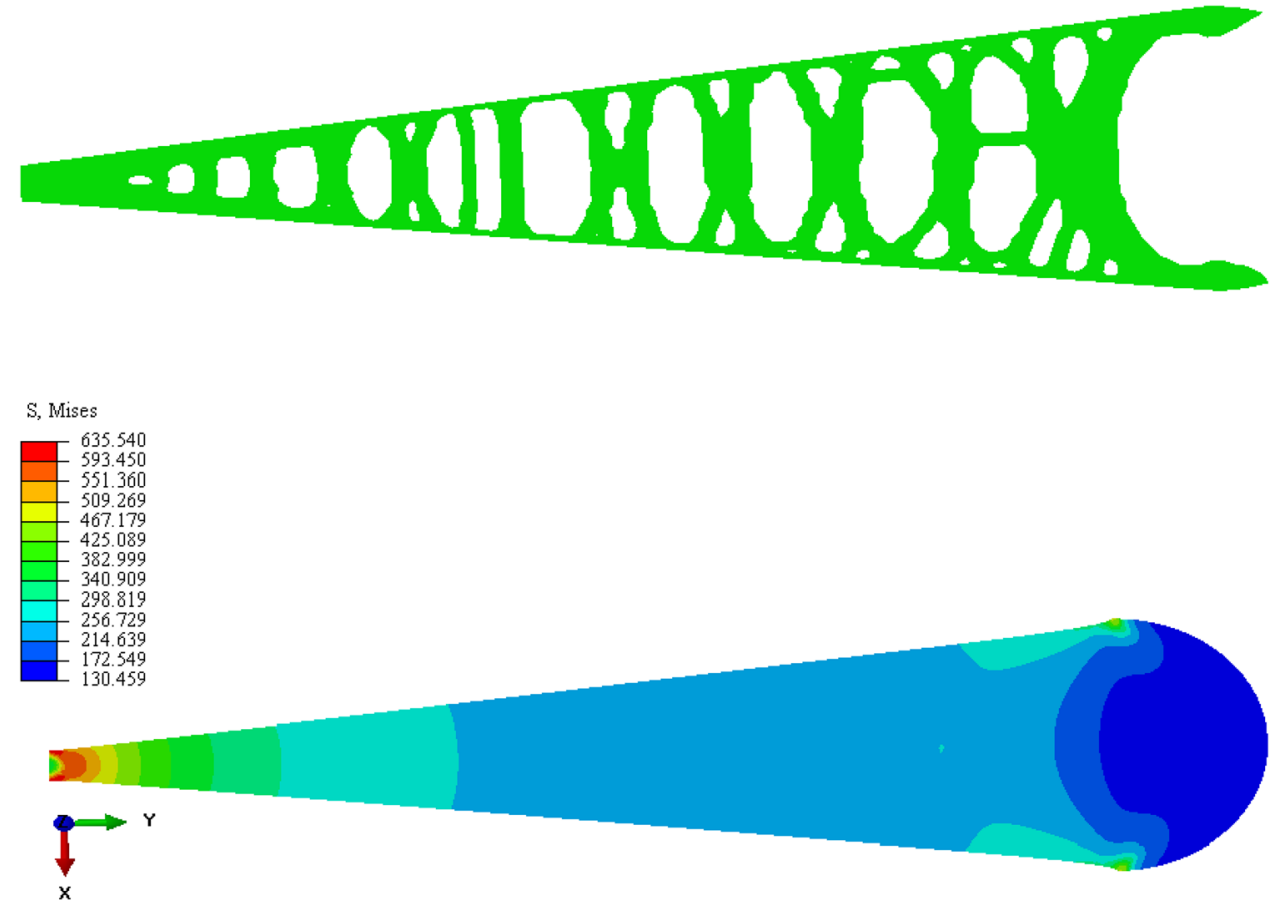
Fig. 17 Stress distribution of the optimum stiffened structure

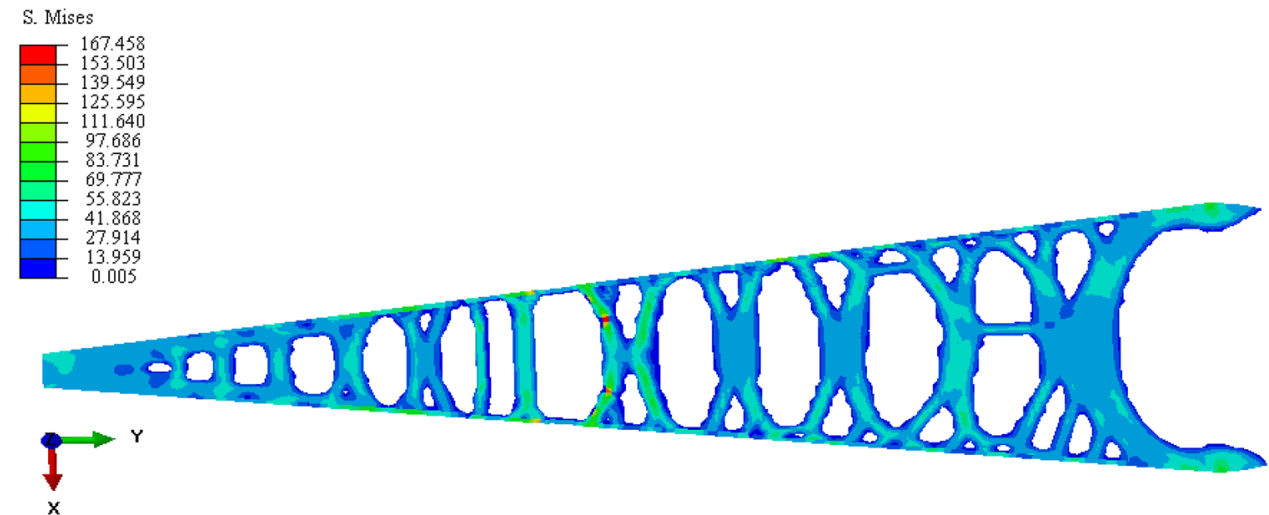

topology optimization. Therefore, we think that the integral composite wing with stiffeners could be easily realized and widely used.

\section{Conclusions}

The present investigations focus on the methodology development of the optimal design for stiffened composite wing panels based on the planning of experiments and emphasize on the close conformity of the developed finite element analysis for an unmanned flat wing micro-aircraft. The above FEA and experimental results have shown that the bending stiffness and bending behaviour are strongly influenced by the stiffener construction of sandwich structure wing panels. The combinatorial stiffener for the wing panel has a higher bending stiffness successively than I-beam, C-beam, skin-stringer and skin-foam structures. The analytical expression of bending stiffness of the sandwich structures for integral wing panel is determined and integrated in terms of the equivalent moment of inertia $I_{s}^{*}$ and profile area $A_{s}{ }^{*}$. This gives the properly standard to measure the optimal solutions obtained for the integral structure wings with different type of stiffeners. It is also validated by combining experimental data with FEM results and a good consistent between them is presented.

The present study shows that an optimal layout of stiffeners can be determined for the integral structure wing to obtain the maximum bending stiffness without weight penalty. Based on the soft-kill BESO method, topology optimization of the wing cross-section is implemented to remove the inefficient materials from the structure. Comparison of the stress and bending stiffness before and after optimization shows that the maximum principle stress reduces from 635.54 to $167.45 \mathrm{MPa}$ under imposing force of $200 \mathrm{~N}$ and the bending stiffness reaches up to $502.9 \mathrm{~N} /$ $\mathrm{mm}$. The colored stress patterns demonstrate that there is no apparent stress concentration occurred in the optimal layout of the structure and the homogeneous stress distribution has revealed the effectiveness of topology optimization in reducing stress concentration and improving load carrying capacity of wing panels.

Acknowledgements The authors would like to acknowledge the support by the National Natural Science Foundation of China (NSFC) (No. 51575189) and China Postdoctoral Science Foundation (No. 2017M610527). We also would like to acknowledge the support by SINOPEC LuoYang Engineering Co., LTD.

\section{Compliance with ethical standards}

Conflict of interest We declare that we have no financial and personal relationships with other people or organizations that can inappropriately influence our work, there is no professional or other personal interest of any nature or kind in any product, service and/ or company that could be construed as influencing the position presented in, or the review of, the manuscript entitled "Structural Design of Composite Stiffened Panel for a Flat Wing Micro-aircraft".

\section{References}

1. Zhang J, Supernak P, Mueller-Alander S, Wang CH (2013) Improving the bending strength and energy absorption of corrugated sandwich composite structure. Mater Des 52:767-773

2. Mouritz AP, Bannister MK, Falzon PJ, Leong KH (1999) Review of applications for advanced three-dimensional fibre textile composites. Compos Part A 30(12):1445-1461

3. Chintapalli S, Elsayed MSA, Sedaghati R, Abdo M (2010) The development of a preliminary structural design optimization method of an aircraft wing-box skin-stringer panels. Aerosp Sci Technol 14(3):188-198

4. Barkanov E, Ozolin O, Eglitis E, Almeida F, Bowering MC, Watson $G$ (2014) Optimal design of composite lateral wing upper covers. Part I: Linear buckling analysis. Aerosp Sci Technol 38:1-8

5. Zhao Q, Ding YL, Jin HB (2011) A layout optimization method of composite wing structures based on carrying efficiency criterion. Chin J Aeronaut 24:425-433

6. Herencia JE, Weaver PM, Friswell MI (2007) Optimization of long anisotropic laminated fibre composite panels with T-shaped stiffeners. AIAA J 45(10):2497-2509

7. Todoroki A, Sekishiro M (2008) Modified efficient global optimization for a HAT-stiffened composite panel with buckling constraint. AIAA J 46(9):2257-2264 
8. Kassapoglou C (1997) Simultaneous cost and weight minimization of composite-stiffened panels under compression and shear. Compos Part A 28(5):419-435

9. Kassapoglou C, Dobyns AL (2001) Simultaneous cost and weight minimization of postbuckled composite panels under combined compression and shear. Struct Multidiscip Optim 21(5):372-382

10. Walker M (2002) The effect of stiffeners on the optimal ply orientation and buckling load of rectangular laminated plates. Comput Struct 80:2229-2239

11. Liu W, Butler R, Mileham AR, Green AJ (2006) Bi-level optimization and post-buckling of highly strained composite stiffened panels. AIAA J 44(11):2562-2570

12. Guo SJ (2007) Aeroelastic optimization of an aerobatic aircraft wing structure. Aerosp Sci Technol 11(5):396-404

13. Monroy Aceves C, Sutcliffe MPF, Ashby MF, Skordos AA, Rodríguez RC (2012) Design methodology for composite structures: a small low air-speed wind turbine blade case study. Mater Des 36:296-305

14. Kim T, Lim J, Shin SJ, Kim DH (2013) Structural design optimization of a tiltrotor aircraft composite wing to enhance whirl flutter stability. Compos Struct 95:283-294

15. Oktay E, Akay HU, Merttopcuoglu O (2011) Parallelized structural topology optimization and CFD coupling for design of aircraft wing structures. Comput Fluids 49:141-145

16. López C, Baldomir A, Hernández S (2016) Deterministic versus reliability-based topology optimization of aeronautical structures. Struct Multidiscip Optim 53(4):907-921

17. Liu Q, Jrad M, Mulani SB, Kapania RK (2016) Global/local optimization of aircraft wing using parallel processing. AIAA J 54(11):3338-3348

18. De S, Jrad M, Kapania RK (2018) Structural optimization of internal structure of aircraft wings with curvilinear spars and ribs. J Aircr 56:1-12

19. Suresh Kumar R, Ray MC (2012) Active constrained layer damping of smart laminated composite sandwich plates using 1-3 piezoelectric composites. Int J Mech Mater Des 8:197-218
20. Allen $H$ (1969) Analysis and design of structural sandwich panels. Pergamon Press, Oxford

21. Li ZB, Zheng ZJ, Yu JL, Qian CQ, Lu FY (2014) Deformation and failure mechanisms of sandwich beams under three-point bending at elevated temperatures. Compos Struct 111:285-290

22. Chen LF, Morozov EV, Shankar K (2012) A combined elastoplastic damage model for progressive failure analysis of composite materials and structures. Compos Struct 94(12):3478-3489

23. Loos AC, Springer GS (1983) Curing of epoxy matrix composites. J Compos Mater 17:135-169

24. Xie YM, Steven GP (1993) A simple evolutionary procedure for structural optimization. Comput Struct 49(5):885-896

25. Querin OM, Young V, Steven GP, Xie YM (2000) Computational efficiency and validation of bi-directional evolutionary structural optimization. Comput Methods Appl Mech Eng 189(2):559-573

26. Yang XY, Xie YM, Steven GP (2005) Evolutionary methods for topology optimization of continuous structures with design dependent loads. Comput Struct 83(12-13):956-963

27. Huang X, Xie YM (2007) Convergent and mesh-independent solutions for the bi-directional evolutionary structural optimization. Finite Elem Anal Des 43(14):1039-1049

28. Liu J, Wen GL, Xie YM (2016) Layout optimization of continuum structures considering the probabilistic and fuzzy directional uncertainty of applied loads based on the cloud model. Struct Multidiscip Optim 53:81-100

Publisher's Note Springer Nature remains neutral with regard to jurisdictional claims in published maps and institutional affiliations. 